\title{
Flaring vs. self-shadowed disks: The SEDs of Herbig Ae/Be stars
}

\author{
C. P. Dullemond ${ }^{1}$ and C. Dominik ${ }^{2}$ \\ 1 Max Planck Institut für Astrophysik, PO Box 1317, 85741 Garching, Germany \\ 2 Sterrenkundig Instituut “Anton Pannekoek”, Kruislaan 403, 1098 SJ Amsterdam, The Netherlands \\ e-mail: dominik@science.uva.nl
}

Received 11 June 2003 / Accepted 19 December 2003

\begin{abstract}
Isolated Herbig Ae stars can be divided into two groups (Meeus et al. 2001): those with an almost flat spectral energy distribution in the mid-infrared ("group I"), and those with a strong decline towards the far-infrared ("group II"). In this paper we show that the group I vs. II distinction can be understood as arising from flaring vs. self-shadowed disks. We show that these two types of disks are natural solutions of the 2D radiation-hydrostatic structure equations. Disks with high optical depth turn out to be flaring and have a strong far-IR emission, while disks with an optical depth below a certain threshold drop into the shadow of their own puffed-up inner rim and are weak in the far-IR. In spite of not having a directly irradiated surface layer, self-shadowed disks still display dust features in emission, in agreement with observations of group II sources. We propose an evolutionary scenario in which a disk starts out with a flaring shape (group I source), and then goes through the process of grain growth, causing the optical depth of the disk to drop and the disk to become self-shadowed (group II source). We show that this scenario predicts that the (sub-)millimeter slope of the disk changes from steep (small grains) to Rayleigh-Jeans-like (large grains) in the early stages of evolution, so that all group II sources are expected to have Rayleigh-Jeans-like slopes, while some group I sources may still have steep (sub-)millimeter slopes.
\end{abstract}

Key words. accretion, accretion disks - stars: circumstellar matter - stars: formation - stars: pre-main-sequence infrared: stars

\section{Introduction}

Herbig $\mathrm{Ae} / \mathrm{Be}$ stars are thought to be the intermediate mass counterparts of T Tauri stars (see e.g. Waters \& Waelkens 1998). They have a strong infrared (IR) excess arising from warm circumstellar dust. Similar to T Tauri stars, this dust is believed to reside in a circumstellar disk. Although there is mounting evidence for the disk-like distribution of this material, this issue is still not completely settled. More importantly, the physics and geometry of such disks are not yet fully understood.

A number of Herbig Ae/Be stars have been studied by Meeus et al. (2001). They present ISO spectra combined with ground-based photometry of 13 Herbig Ae/Be stars, and convincingly show that they can be classified into two main groups, one of which can be subdivided into two more subgroups. The main division (group I vs. II) distinguishes the sources on the basis of the shape of the overall spectral energy distribution (SED). Group I sources have a relatively strong far-IR flux, which is energetically comparable with the flux in the near-IR. Group II sources show a similar near-IR excess as group I sources, but their flux falls off strongly towards the far-IR. The fraction of energy reprocessed by circumstellar

Send offprint requests to: C. P. Dullemond, e-mail: dullemon@mpa-garching.mpg.de dust is typically $30-50 \%$ in group I sources and about $15 \%$ to $30 \%$ in group II sources. Examples of these two different kinds of SEDs are shown in Fig. 1.

In the paper of Meeus et al. it was speculated that the distinction between group I sources and group II sources might be explained qualitatively by the disk having a flaring geometry or not. A flaring disk captures and reprocesses more stellar radiation at large radii than flat disks do, and therefore naturally has a stronger far-infrared excess. However, it remained unclear what physical mechanism could cause some disks to be flaring and others to be flat. In fact, models of passive circumstellar disks (e.g. D'Alessio et al. 1998; Chiang \& Goldreich 1997, CG97; Dullemond et al. 2001, DDN01) have so far shown that these disks consistently have a flaring geometry.

A possible mechanism that could flatten a disk is dust settling. Due to the gravity exerted by the central star, dust grains can slowly drift towards the midplane of the disk while the gas remains behind. Since the stellar radiation is absorbed almost exclusively by dust, the photosphere of the disk flattens as dust settling proceeds. This mechanism has been proposed by Chiang et al. (2001) as an explanation for the mid- and farIR SEDs of the Herbig stars MWC 480 and HD 36121. They found that the SEDs of these sources could only be fitted with a CG97 type disk if the vertical geometric thickness was artificially reduced by a factor of about 3 to 5 . They argue that this 


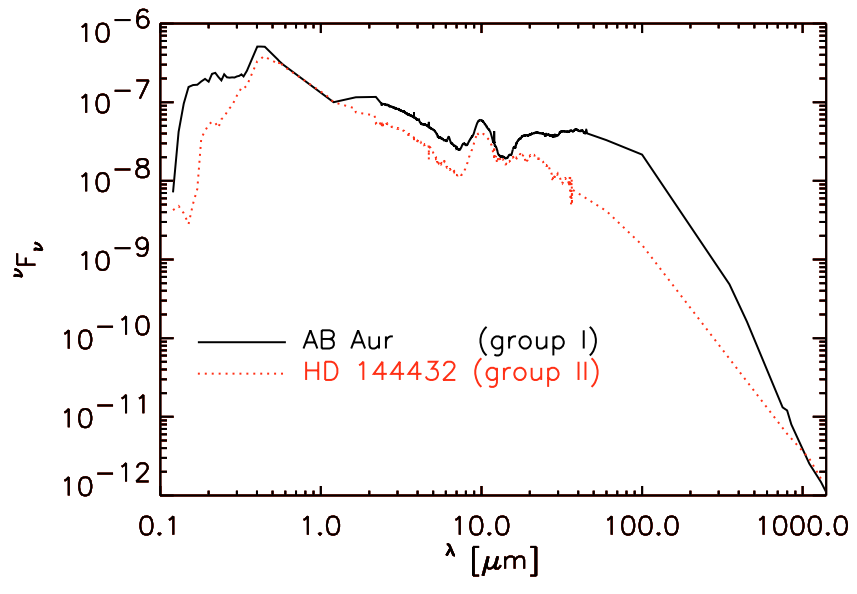

Fig. 1. Two examples of SEDs of Herbig Ae stars of the sample of Meeus et al. showing the qualitative difference in the SEDs of group I sources and group II sources.

suggests that dust settling is the mechanism behind the group II type SEDs.

In this paper we will demonstrate a more direct, though possibly related, way of causing a disk to have a group II type SED. It is the effect of self-shadowing. If the disk's geometric thickness $H / R$ is a monotonic increasing function of radius $R$, then the disk is flaring, and can capture stellar radiation at all radii. If, on the other hand, the ratio $H / R$ becomes smaller as one goes to larger radii, then the disk becomes self-shadowed: the inner disk regions cast a shadow over the outer disk regions. As the shadow deprives the outer regions from most of their irradiation flux (only indirect irradiation remains possible, but is rather weak), the far-IR emission is strongly suppressed. This works even without the need of a factor of 3 to 5 reduction in $H$, as long as the outer parts of the disk are in the shadow of the inner parts. Dust settling could be a possible mechanism causing such a shadowing (Dullemond \& Dominik in prep.), but another possibility is the vertical optical depth of the disk, as we will show in this paper.

We will show that an important role in the self-shadowing is played by the disk's puffed up inner rim. The existence of such a structure was inferred from observations of Herbig $\mathrm{Ae} / \mathrm{Be}$ stars in the near-IR. Virtually all Herbig Ae/Be stars show a prominent thermal bump in their SEDs around $3 \mu \mathrm{m}$. Since a simple flaring disk model is not capable of reproducing it, several authors invoke additional non-disk components to explain this feature (Miroshnichenko et al. 1997, 1999; van den Ancker et al. 2000). But Natta et al. (2001) have drawn attention to the importance of the inner hole in the disk caused by dust evaporation, and the emission from the inner rim of the dusty part of the disk. It was shown by Dullemond et al. (2001, henceforth DDN01) that the model of Chiang \& Goldreich, extended by a self-consistent (though simplified) description of the inner rim, could indeed explain the entire SEDs of Herbig Ae stars, in particular those of group I. An important feature of these models is that the inner rim of the dusty disk is puffed-up as a result of its direct exposure to the light of the central star. The rim therefore casts a shadow over part of the disk, typically from the location of the $\operatorname{rim}(\sim 0.5 \mathrm{AU})$ out to about $\sim 5 \mathrm{AU}$.
Only further out the surface height of the disk becomes sufficiently high to cause the upper layers of the disk to reach out of the shadow wedge cast by the inner rim (see Fig. 8 left).

While the DDN01 models work best for group I sources, they can also fit group II sources (even without dust settling). But these fits require an unlikely termination of the disk just outside of the shadow cast by the inner rim (Dominik et al. 2003). It seems unlikely that all group II sources have such a fine-tuned outer radius. For some sources it is also known from other observations that the disks are much larger (Mannings \& Sargent 1997). Dominik et al. argue that the inferred smallness of the flaring part of the disk may in fact be a hint that these disks are fully self-shadowed, i.e. that the inner rim shadows the entire disk. However, no detailed models of such self-shadowed disks were given.

A consistent discription of self-shadowed (non-flaring) disks can only be achieved by employing a 2D (axisymmetric) or $3 \mathrm{D}$ radiative transfer code to compute the temperature structure of the disk. By also computing the vertical density structure of the disk simultaneously, it can be investigated if, and under what circumstances non-flaring or self-shadowed disks can exist. A first attempt in this direction was done by Dullemond (2002, henceforth D02). Using a grey 2D radiative transfer code it was shown that disks with a high optical depth flare in the way described by DDN01 (inner rim, shadow and outer flaring part). But when the optical depth of the disk is below a certain threshold, the entire disk drops into the shadow of its own inner rim (see Fig. 8 right). The resulting SED turns out to be very similar to group II sources: a strong near IR bump and a weak far-IR flux with a steep slope. In the D02 paper it was therefore suggested that the group II type of SED is caused by self-shadowing, instead of a mere flattening of a flaring disk.

In spite of these new findings, a number of important questions remain to be answered before firm conclusions can be drawn. In particular, the assumption of grey opacities in D02 is very unrealistic. If the disk is dominated by small grains (as indicated by the prominent emission features) the opacity is far from grey. It is possible that real opacities will change the picture considerably. Moreover, with real opacities we can compare the model to the observations in more detail, and we can assign realistic masses to our disk models where previously we could only speak of the optical depth. Therefore, the present paper addresses the following issues:

- Does the group I/II explanation in terms of flaring/selfshadowed still hold when real opacities are used? If so, for which disk parameters do we get flaring or self-shadowed disks? Does dust grain growth play a role in the division between group I and group II sources?

- Will self-shadowed disks provide the 10 micron feature in emission, as seen in most group II sources? In the simple picture of a CG97 or DDN01 disk, these features are always formed in the superheated layer created by dust directly illuminated by the star. In a self-shadowed disk the disk surface is not directly irradiated.

- What do the models predict for the (sub-)mm flux and slope for group I and group II disks? The grey models were 
entirely optically thick in the (sub)mm, which may not be the case when real opacities are used.

In this paper we present the first self-consistent $2 D$ models of Herbig Ae star disks with realistic dust opacities, and we show that indeed the discrepancy between group I and group II sources can be understood naturally in terms of flaring and selfshadowed (i.e. non-flaring) disks. We also show that through the reduction of the optical depth of the disk by the growth of grains, a flaring disk can be turned into a self-shadowed disk. This suggests a natural evolutionary link between group I and group II sources.

The paper is organized as follows: in Sect. 2 we describe the modeling procedure and radiative transfer codes used. In Sect. 3 we describe the structure of - and the SEDs produced by - two sets of models which run from large to low optical depth of the outer disk parts. In Sect. 4 we discuss the results in the framework of shadowing, rim emission, solid state features and grain growth.

\section{Models}

\subsection{Modeling procedure}

The equations for the models of this paper are very similar to the ones presented in D02, but while in D02 a grey opacity is used for the sake of simplicity, we now use silicate grains with a size of $a=0.1 \mu \mathrm{m}$ (Draine \& Lee 1984). In order not to complicate the models unnecessarily we ignore the scattering opacity (we set it to zero). An extensive discussion of the effects of scattering in models of passive reprocessing disks is given in Dullemond \& Natta (2003).

To compute the disk structure, we proceed as follows. We adopt as our coordinate system the polar coordinates $R$ and $\Theta$ (where $\Theta=0$ means the pole and $\Theta=\pi / 2$ is the equator), and we assume axial symmetry. The disk is presumed to be passive, i.e. non-accreting, so that the energy balance is entirely determined by the irradiation by the central star. This process of irradiation is modeled using a 2D axisymmetric continuum radiative transfer code that solves for the dust temperature as a function of $R$ and $\Theta$.

The determination of the density structure requires an iteration procedure. An input to the model is the surface density distribution $\Sigma(R)$. At the start we set up a reasonable initial guess for the density distribution $\rho(R, \Theta)$ that is consistent with this $\Sigma(R)$. We can then apply the radiative code to find the gas temperature everywhere. Using this temperature distribution, a vertical integration of the equation of vertical hydrostatic equilibrium yields the new 2D density structure $\rho(R, \Theta)$. After iterating the entire procedure of radiative transfer and vertical structure a couple of times one finds a solution in which both $\rho(R, \Theta)$ and $T(R, \Theta)$ are consistent with each other. Using this density and temperature structure a ray-tracer can now produce the required SEDs and images.

There is, however, a major hurdle that needs to be overcome before the above schetched procedure can work. The disks that we wish to model may have tremendous optical depths. An optical depth of $\tau_{V}=10^{5}$ is not uncommon. Most radiative transfer codes cannot handle such optical depths: they will either produce noisy or wrong results, or they never converge. As was described in D02, to solve this problem we use the code RADICAL, which is based on the method of Variable Eddington Tensors (VET). This method is the only method presently known to be able to treat problems with such enormous optical depth without requiring excessive execution time. For $1+1 \mathrm{D}$ type disk models they have been succesfully applied many times already (see, e.g., Malbet et al. 1991, 2001; Dullemond et al. 2002). For 2D radiative transfer problems they are slowly coming in use (e.g. Nakazato et al. 2003; D02).

The problem with the VET algorithm implemented in RADICAL is that it sometimes has slight inaccuracies in the resulting temperature profiles. These inaccuracies are usually not larger than about $5 \%$ to $10 \%$, but since the SED is proportional to $T^{4}$, such inaccuracies are amplified in the outcoming spectrum. For the structure iteration these inaccuracies are less dangerous, as the pressure scale height of the disk is proportional to $\sqrt{T}$, meaning that the error in the density structure of the disk is $2.5 \%$ to $5 \%$, which is no cause for alarm. It seems therefore that RADICAL is appropriate for the structure iteration procedure, but not for computing the temperature profile for the ray-tracer producing the SED and images.

For the final temperature structure determination (after convergence of the density structure) we therefore prefer to use a different method: an improved version of the algorithm of Bjorkman \& Wood (2001), implemented in a code called RADMC. As expected, this method suffers from large CPU costs at high optical depth, and in many cases produces an strong numerical noise on the temperature profile in the most optically thick regions. But this is not a major problem since these optically thick regions (usually near the equator at small radii) are unobservable anyway. And since RADMC is called only once per model (while RADICAL has to be called many times during the structure iteration), the large CPU cost of RADMC remains manageable. RADMC has the advantage of being very accurate and reliable when it comes to the emerging spectrum. We have found that for some of the models RADICAL slightly overpredicts the 5-8 $\mu \mathrm{m}$ part of the SED, while RADMC produces a sharper transition between the mid-infrared-bump and the 10-micron feature, in agreement with the SEDs predicted by semi-analytic models (Dullemond et al. 2001), and found in nature (e.g. Meeus et al. 2001). Since the Bjorkman \& Wood method is a statistical simulation of the motion of photons through the disk, the RADMC code is more reliable when it comes to exact spectral shape than the RADICAL code. To be sure that the use of RADICAL for the structure iteration is nevertheless justified, we verified that the density structure produced after the RADMC run is virtually identical to the one produced with the RADICAL code, for those cases in which the RADMC code could produce low-noise temperature profiles (typically the lesser optically thick cases). Also, both codes have been independently tested against other codes on a number of test cases, albeit of much less extreme optical depths than here (Pascucci et al. 2004). Both the RADICAL results and the RADMC results conserve energy to within an error of at most $5 \%$.

As final note it should be mentioned that the iteration procedure, in which the hydrostatic equilibrium code and the 
radiative transfer code are alternately applied, may in some cases not reach a perfectly converged solution. Minor waves may propagate over the solution from one iteration to the next and never damp out completely. This problem is related to a known instability operating in these disks (Dullemond 2000) and may point to such waves propagating the disk in reality as well. For disks around Herbig Ae/Be stars, like the models presented in this paper, these waves are weak and have virtually no effect on the SED or images. For T Tauri stars the problem is more serious, and a study of the stability of these disks using time-dependent heating and cooling calculations is required. For this reason we limit ourselves in this paper to Herbig Ae/Be stars only.

\subsection{Setup of the models}

The models of this paper simulate a disk around a star of $M_{*}=$ $2.5 M_{\odot}, R_{*}=2 R_{\odot}$ and $T_{*}=10000 \mathrm{~K}$. The surface density of the disk as a function of radius $\Sigma(R)$ is defined to be:

$\Sigma(R)=\Sigma_{0}\left(R / R_{0}\right)^{p}$

with $R_{0}$ taken to be $R_{0}=200 \mathrm{AU}$. The mass of the disk is then:

$M_{\text {disk }}=\left\{\begin{array}{l}2 \pi \Sigma_{0} R_{0}^{-p} \frac{1}{p+2}\left[R_{\text {out }}^{p+2}-R_{\text {in }}^{p+2}\right](\text { for } p \neq 2) \\ 2 \pi \Sigma_{0} R_{0}^{-p}\left[\ln R_{\text {out }}-\ln R_{\text {in }}\right](\text { for } p=2) .\end{array}\right.$

Similar equations hold for the dust mass of the disk $M_{\text {dust }}$ in relation to the dust surface density $\Sigma_{\text {dust }}$. In general one has $M_{\text {dust }}=0.01 M_{\text {disk }}$ and $\Sigma_{\text {dust }}=0.01 \Sigma_{\text {disk }}$, because of the gas-to-dust ratio of 100 .

We take the inner radius to be at $0.5 \mathrm{AU}$, which is approximately the dust evaporation radius, and we put the outer radius at $200 \mathrm{AU}$. We allow the disk to extend a bit further than $200 \mathrm{AU}$, but with a very steep powerlaw index for the surface density $\Sigma \propto R^{-12}$, so that effectively the disk has a is 200 AU outer radius, but does not end too abruptly there.

In this paper we present three series of models:

- Series A: disks with equal mass $\left(M_{\text {disk }}=0.01 M_{\odot}\right.$, i.e. $\left.M_{\text {dust }}=0.0001 M_{\odot}\right)$, but different distribution of $\Sigma(R)$ (power law index between $p=-1 \cdots-4$ ). The grain size is $0.1 \mu \mathrm{m}$.

- Series B: disks with varying mass (between $M_{\text {disk }}=$ $0.1 \cdots 10^{-6} M_{\odot}$, i.e. $\left.M_{\text {dust }}=10^{-3} \cdots 10^{-8} M_{\odot}\right)$, but with the same distribution of this mass as a function of radius $(p=-1.5)$. The grain size is again $0.1 \mu \mathrm{m}$.

- Series BL: like the B series, but the reduction in mass of the disk is now a reduction only in the small grain dust mass $(0.1 \mu \mathrm{m})$. As the mass in small grains is reduced, the removed dust mass is converted into a layer of large (2 $\mathrm{mm})$ grains at the equatorial plane, so that the total dust mass $(0.1 \mu \mathrm{m}$ and $2 \mathrm{~mm}$ grains $)$ remains the same $\left(M_{\text {dust }}=0.001 M_{\odot}\right)$. In this way we simulate the process of grain growth, and the subsequent settling of the large grains to the midplane. The small grains are assumed not to have settled.

The parameters of all these models are listed in Table 1.
Table 1. The parameters of the three series of models presented in this paper. $p$ is the power law index for the surface density $\left(\Sigma(R) \propto R^{p}\right)$. $M_{\text {dust,small }}$ is the mass in small $(0.1 \mu \mathrm{m})$ grains. These grains are evenly distributed with the gas in a constant gas-to-dust mass ratio. For the $\mathrm{A}$ and $\mathrm{B}$ models, this ratio is 100 . For the BL models this ratio is 100, 1000, 10000 etc., but still constant over the disk. The $M_{\text {dust,big }}$ is the mass in big $(2 \mathrm{~mm})$ grains which are assumed to be located in a thin midplane layer. This parameter is only non-zero for the BL series, and it is taken such that $M_{\text {dust,small }}+M_{\text {dust,big }}=10^{-3} M_{\odot}$. In this way, the BL series simulates a process of converting small grains evenly distributed in the disk into big grains located at the midplane. $M_{\text {disk }}$ is the total mass of the disk (dust+gas) as calculated from the three disk parameters. It is therefore not a model parameter. Note that the B1 and BL1 models are identical.

\begin{tabular}{c|ccc|c}
\hline \hline & $p$ & $M_{\text {dust,small }} / M_{\odot}$ & $M_{\text {dust,big }} / M_{\odot}$ & $M_{\text {disk }} / M_{\odot}$ \\
\hline A1 & -1 & $10^{-4}$ & 0 & $10^{-2}$ \\
A2 & -2 & $10^{-4}$ & 0 & $10^{-2}$ \\
A3 & -3 & $10^{-4}$ & 0 & $10^{-2}$ \\
A4 & -4 & $10^{-4}$ & 0 & $10^{-2}$ \\
\hline B1 & -1.5 & $10^{-3}$ & 0 & $10^{-1}$ \\
B2 & -1.5 & $10^{-4}$ & 0 & $10^{-2}$ \\
B3 & -1.5 & $10^{-5}$ & 0 & $10^{-3}$ \\
B4 & -1.5 & $10^{-6}$ & 0 & $10^{-4}$ \\
B5 & -1.5 & $10^{-7}$ & 0 & $10^{-5}$ \\
B6 & -1.5 & $10^{-8}$ & 0 & $10^{-6}$ \\
\hline BL1 & -1.5 & $10^{-3}$ & 0 & $10^{-1}$ \\
BL2 & -1.5 & $10^{-4}$ & $9.0 \times 10^{-4}$ & $10^{-1}$ \\
BL3 & -1.5 & $10^{-5}$ & $9.9 \times 10^{-4}$ & $10^{-1}$ \\
BL4 & -1.5 & $10^{-6}$ & $9.99 \times 10^{-4}$ & $10^{-1}$ \\
BL5 & -1.5 & $10^{-7}$ & $1.0 \times 10^{-3}$ & $10^{-1}$ \\
BL6 & -1.5 & $10^{-8}$ & $1.0 \times 10^{-3}$ & $10^{-1}$ \\
\hline
\end{tabular}

The A series allows us to see whether self-shadowed disks can result from redistributing matter in the disk from the outside to the inside. The B series shows whether an overall reduction of the optical depth (i.e. mass) can cause self-shadowing. The BL series is like the B series, but simulates grain growth and settling (see D'Alessio et al. 2001 for an earlier study of the effect of grain growth on disk models).

\section{Results}

\subsection{Structure of the disk}

To show the structure of the computed models, we use contours of temperature and density in a plot in which the 2-dimensional spherical coordinates $\log (R)$ and $\pi / 2-\Theta$ are on the $x$ - and $y$-axes respectively. The midplane is at the bottom of the figure $(\theta=\pi / 2)$. These plots show the entire disk structure in a clear way. Lines at constant $\pi / 2-\Theta$ (radial rays) are horizontal lines in this plot. Flaring and non-flaring disks can therefore easily be distinguished by plotting the $\tau=1$ location along these lines: flaring disks will be indicated by rising 

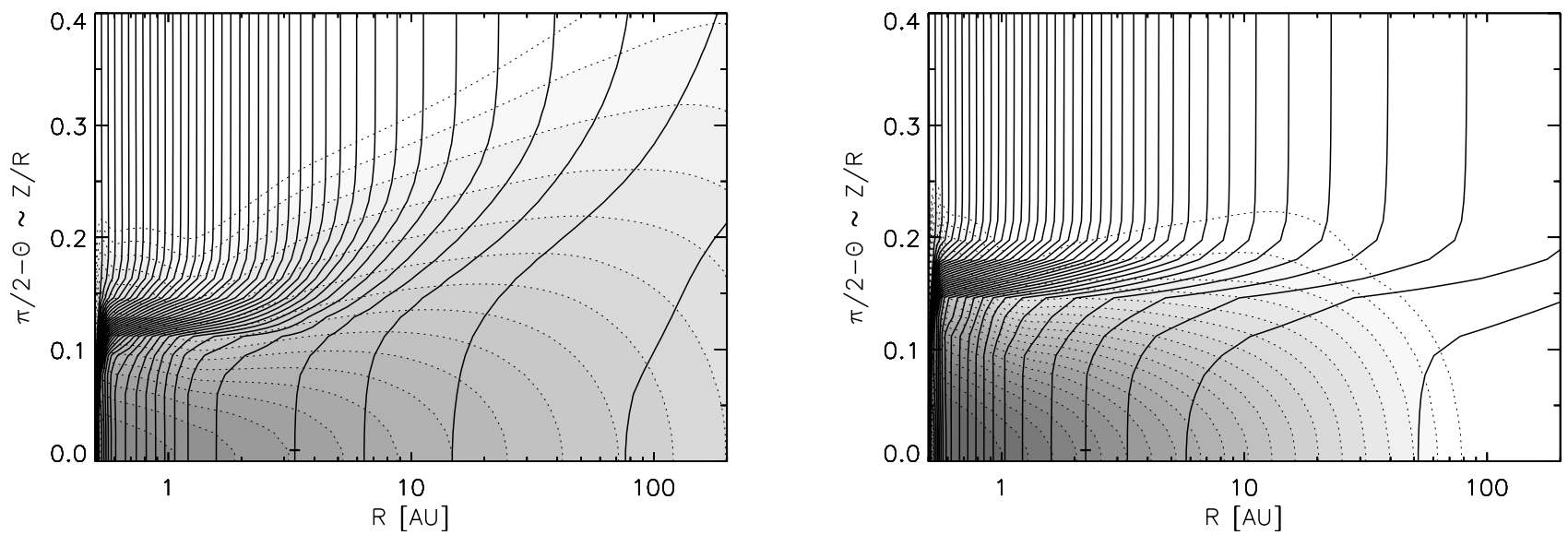

Fig. 2. The temperature and density structure as a function of spherical radius $r$ and polar angle $\theta(\pi / 2$ is the equatorial plane, i.e. the bottom of the figure) for model A1 (left) and A4 (right). The grey-scale contours (accentuated by the dotted contour lines) are density. These are spaced logarithmically in steps of a factor 3.3. The solid contours are temperature. The temperature contours are spaced $50 \mathrm{~K}$ apart. The small tick mark on one of these contours tags the $200 \mathrm{~K}$ contour.
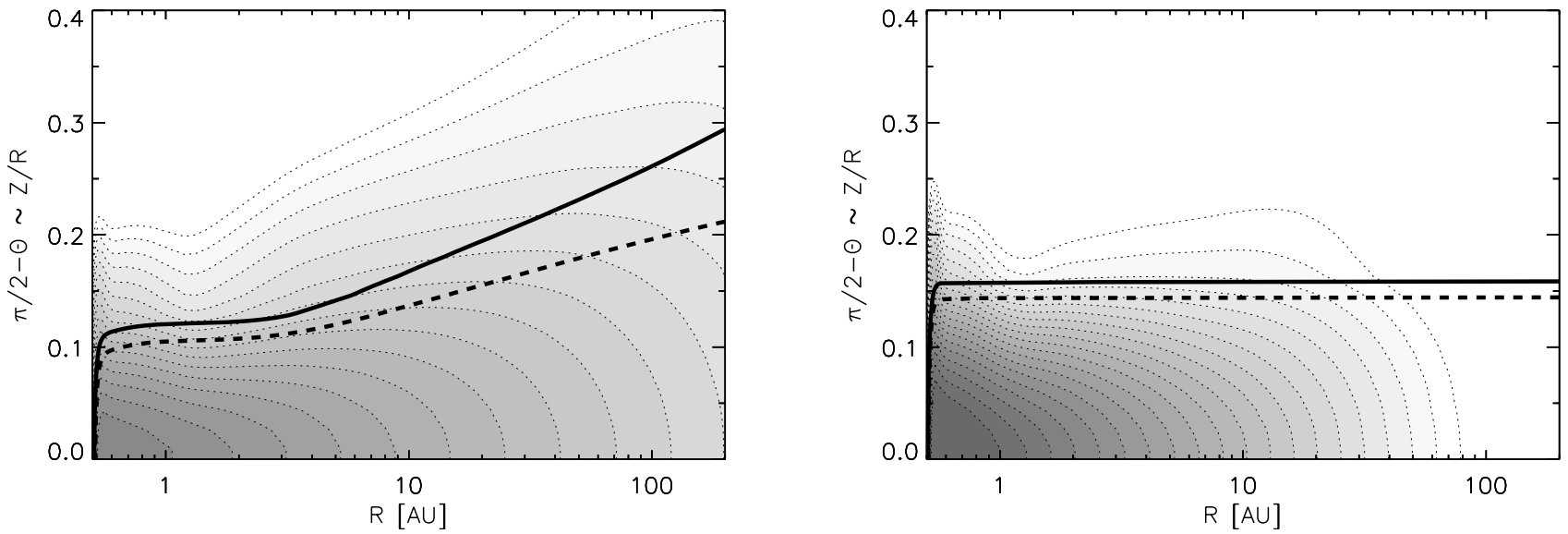

Fig. 3. As Fig. 2, but instead of temperature contours, the figure shows the surface of $\tau_{0.55} \mu \mathrm{m}$,radial $=1$ (solid), and $\tau_{3} \mu \mathrm{m}$,radial $=1$ (dashed). The shape of these curves clearly show that the left model is flared while the right model is self-shadowed. In the flared model it is also seen that the flaring starts for real beyond about $8 \mathrm{AU}$; between 0.5 and $8 \mathrm{AU}$ the curve stays at almost constant $\Theta$, typical for the shadowed region of the disk.

curves while non-flaring disks will show flat curves. The resulting temperature and density structures for models A1 and A4 are shown in Fig. 2 in the way explained above. In Fig. 3 the same models are shown, but this time only the density contours. Overplotted are the $\tau=1$ surfaces at $0.55 \mu \mathrm{m}$ and $3 \mu \mathrm{m}$. In these figures one can clearly see the puffed-up structure of the inner rim in both models, in agreement with the DDN01 model. But at large radii only model A1 has rising density contours, while model A4 has constant or even declining density contours. This difference is typical of the the difference between flaring and self-shadowed disks. The distinction is more clear in the shape of the $\tau=1$ surfaces: for the flaring model (A1) they move upward toward larger radius, while for the selfshadowed model (A4) they remain at constant $\pi / 2-\theta$. It is interesting to note that for the flaring model (A1) the upward movement of the $\tau=1$ starts around about $3 \mathrm{AU}$, while the curve remains approximately constant below $3 \mathrm{AU}$. This is the shadow of the inner rim that covers the innermost $(<3 \mathrm{AU})$ part of the disk. This phenomenon was predicted by DDN01, and is confirmed here.
The temperature contours of Fig. 2 are somewhat harder to interpret. Clearly the temperature at the disk midplane is lower than the temperature of grains above the disk's surface. This is the reason the disk has dust features in emission. The "kink" in the temperature profile roughly follows the $\tau=1$ surface, since $\tau=1$ defines the surface where the direct stellar radiation is absorbed.

Many of the qualitative structual features of these models are similar to the models of D02, and we refer to that paper for a deeper discussion and interpretation of the temperature and density contours shown here.

The models from series B shows similar results. Figure 4 shows the structure plots of models B1 and B5. Model B1 has the same kind of flaring shape as model A1. As one goes toward lower mass (B5) the disk tends to become more selfshadowed, resembling model A4, but with a less high inner rim. This shows that decreasing the mass of the disk can have a similar effect as changing the power law slope of the disk. But the effect is less abrupt. Model B4, for example, is intermediate between a flaring and a self-shadowed disk, and even model B5 


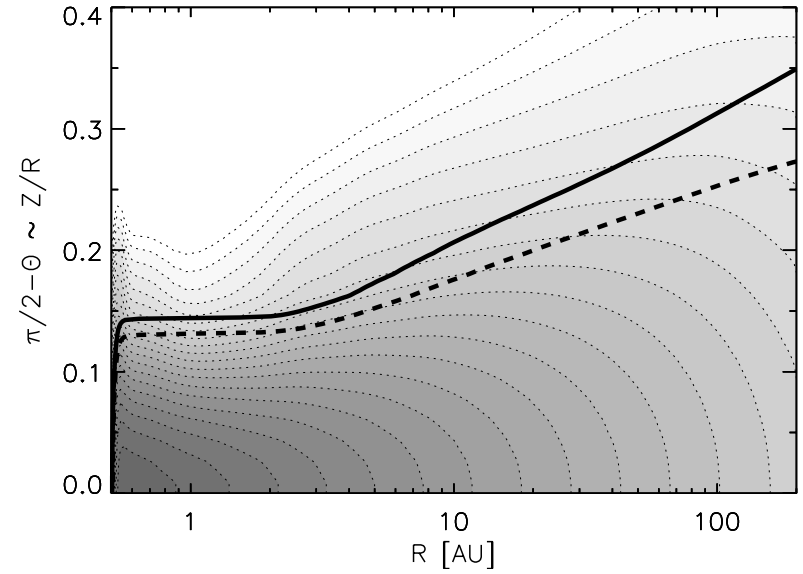

Fig. 4. As Fig. 3, but this time for model B1 (left) and B5 (right).

is less self-shadowed than model A4 (compare the left panels of Figs. 3 and 4). This is because in the B series both the height of the inner rim as well as the height of the surface of the disk behind it get reduced. The reduction of the disk surface height is faster, and that is the reason why from B1 to B6 the flaring disk turns into a self-shadowed one.

We do not show density and/or temperature structure figures for the BL series, since the global structure of the BL disk models is the same as for the B disk models. The only real difference is the presence of the passive midplane layer of large grains in the BL models. These grains do not affect the temperature structure of the disk. The fact that the gas mass of the $\mathrm{BL}$ disks are all identical while for the B series they decrease from B1 to B6 (see Table 1) has no effect on the structure, because the gas-to-dust ratio drops out of the equations for passive disks, as long as it remains a global constant of the model. Therefore, the temperature structure and, except for a global factor, the density structure of the B and BL disk models are the same. In the modeling procedure for the BL series we in fact use the density structure of the corresponding B model, add the midplane layer, do one more radiative transfer run with the RADMC code to compute the midplane grain temperature and finally create the SEDs for the BL series.

\subsection{The SEDs of the disks}

In Fig. 5 the spectral energy distributions of the models of series $A$ are shown. The SED varies from one that has a strong far-IR flux to an SED that is dominated by near-IR flux. The models with a strongly flaring outer disk (models A1, A2) are the ones that have a strong far-IR flux. The radiation of the central star is thereby captured by the disk at large radii, and reprocessed into far-IR emission. In particular in model A1, most of the mass is in the outer regions of the disk. The flaring part has a large covering fraction with respect to the central star, and is therefore bright. Moreover, the low density in the inner rim region weakens the shadow effect, exposes a larger part of the outer disk to stellar radiation and flaring, and reduces the near-IR flux.

The models with a self-shadowed geometry (models A3, A4) turn out to have a low far-IR flux (Fig. 5). A significant

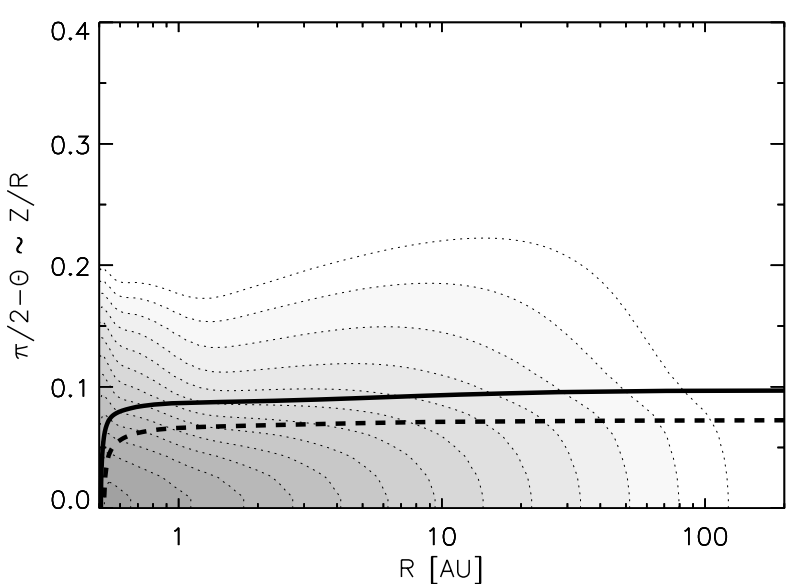

fraction of the disk mass resides in the inner regions, close to the rim. The inner rim is optically thick to large heights and casts a strong shadow over the disk. The mass in the outer regions of the disk is so low that the disk surface stays below the shadow cast by the inner rim. The entire disk is non-flaring (it receives no direct radiation from the star) and falls into the shadow, with indirect radiation from the upper parts of the inner rim as the only remaining heating source. Nevertheless, the silicate feature at $10 \mu \mathrm{m}$ is still in emission, albeit weaker in the A4 model than in the other models.

The SEDs of the B series are shown in Fig. 6. The first five models are still reasonably optically thick, and are representative for Herbig Ae/Be stars. Models B6 starts to become optically thin even at near-IR wavelengths, and therefore represents a transition toward optically thin disks such as the debris disks often found around Vega-type stars. It is clear that the flaring disk models (B1 to B3) still have a reasonably strong far-IR flux while the self-shadowed models (B4 to B6) have a weak far-IR flux. This is consistent with the group I/II distinction. Also, all models have a strong $10 \mu$ silicate feature in emission, even the self-shadowed models (B4 to B6), which is consistent with observations.

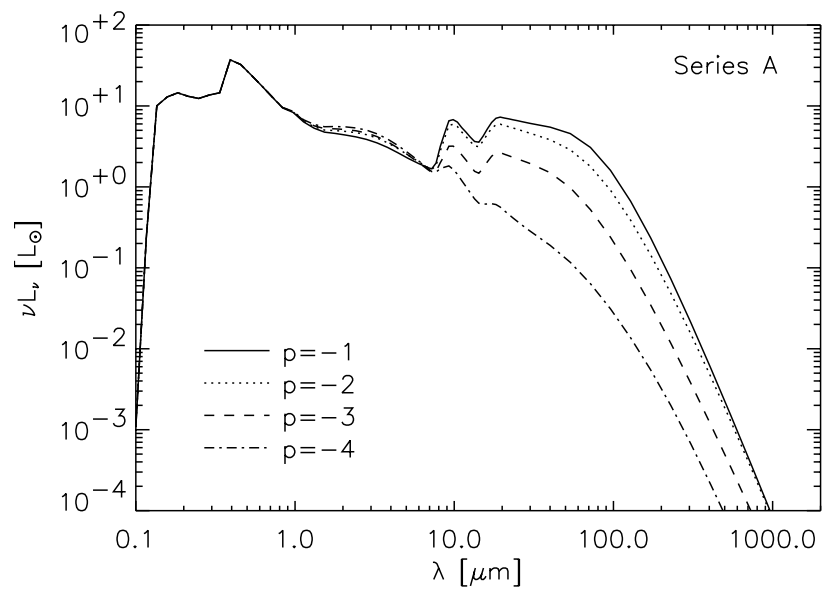

Fig. 5. The SEDs of models A1 ...A4 plotted over each other. The SEDs are at inclination $i=45^{\circ}$. 


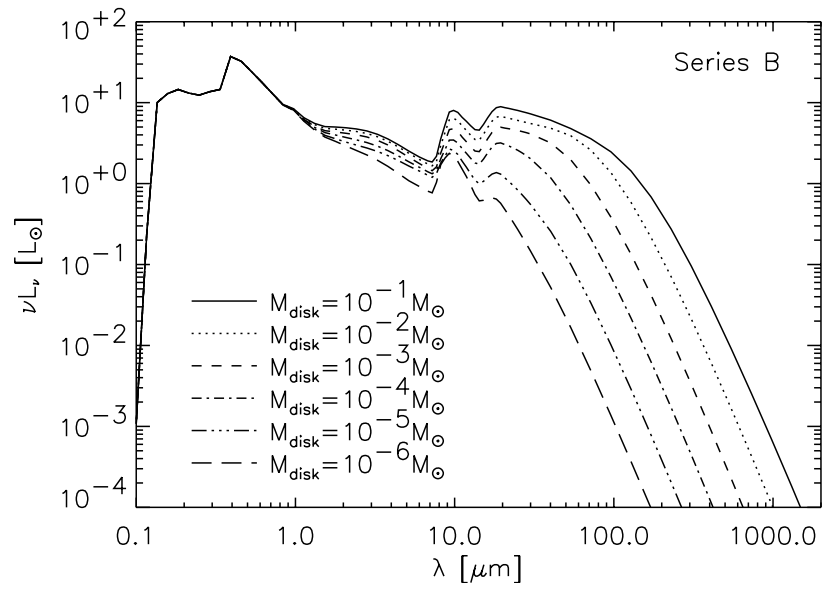

Fig. 6. The SEDs of models B1...B6 plotted over each other. The SEDs are at inclination $i=45^{\circ}$.

Interestingly, the infrared flux at different wavelengths decreases sequentially from model B1 to B6. First the mm flux diminishes, then the far-IR, followed by the mid-IR and finally the near-IR. The near- to far-IR part of this trend seems to be consistent with the observed differences between group I and group II sources. However, the (sub-)mm fluxes of the "group II models" (B4 to B6) are much less than those of real group II sources. In fact, the (sub-)mm fluxes of group II sources are in reality similar to those of group I sources. Therefore, the group I/II distinction can not be explained by merely a distinction in disk mass.

The BL series is similar to the B series but the removed mass in small grains is put into large grains located at the disk's midplane. In Fig. 7 the SEDs of these models are shown. The near- to far-IR behavior of the BL series is the same as the B series. But in the $\mathrm{BL}$ models the midplane layer of large grains keeps the (sub-)mm flux to a certain level, even when the far-IR flux drops from B1 to B6. Therefore, from these results one expects no big systematic differences in the (sub-)mm flux levels between group I and group II sources, which is consistent with observations.

Another striking feature of the BL series is that model BL1 (which only has small grains) has a much steeper (sub-)mm slope than the BL2 to BL6 models. If grain growth and settling governs the transition from group I to group II, we would therefore predict that some (though not all) group I objects have steep (sub-)mm slopes, while all group II sources have less steep (i.e. more Rayleigh-Jeans-like) slopes. This is indeed consistent with observations: group I disks sometimes have steep slopes, indicating that they are dominated by small grains, while group II disks generally have much more shallow slopes indicative of large grains (Bouwman et al. 2000; Acke et al., A\&A subm.).

One thing that is not very well in agreement with observations is that in the B and BL series, as one goes from flaring to self-shadowed, also the flux around $10 \mu \mathrm{m}$ is somewhat suppressed. The ratio of $10 \mu \mathrm{m}$ to $40 \mu \mathrm{m}$ flux does not change too much. In reality there exist many group II sources with a much larger flux ratio between $10 \mu \mathrm{m}$ and the far-IR than these models predict.

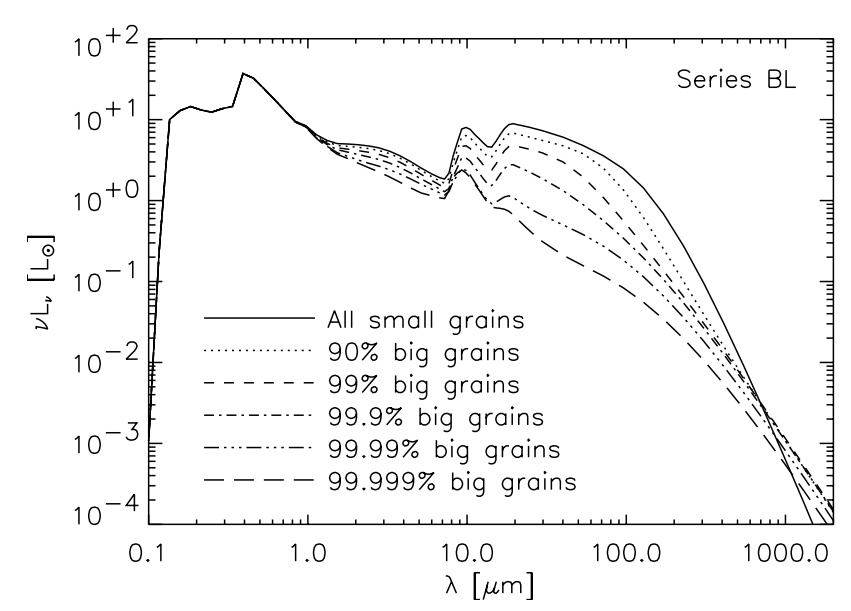

Fig. 7. The SEDs of models BL1...BL6 plotted over each other. The SEDs are at inclination $i=45^{\circ}$.

Nevertheless, the BL series, as a model of the origin of the group I versus group II distinction, seems to be reasonably well in agreement with observations, and may point toward an evolutionary link between these two groups of Herbig $\mathrm{Ae} / \mathrm{Be}$ stars.

\section{Discussion}

\subsection{Flaring versus self-shadowed}

We have found two main types of solutions to the coupled 2D continuum radiative transfer and vertical structure problem for passive irradiated circumstellar disks around Herbig $\mathrm{Ae} / \mathrm{Be}$ stars. Some disks are flared from about $3 \mathrm{AU}$ outwards, and other disks are entirely self-shadowed. A pictographic representation of the two main types of solutions is shown in Fig. 8. The SEDs from these two types of models are very reminiscent of the two types of SEDs observed from Herbig $\mathrm{Ae} / \mathrm{Be}$ stars: those with a strong far-IR flux and those with a weak far-IR flux.

It is not uniquely determined which parameter switches the disk from one mode into the other. Self-shadowing is driven by two main factors: there must be enough matter in the inner rim to produce a shadow, and there must be too little mass at large radii to keep the disk's surface above the shadow. The first condition is almost always met: already for very little matter near the inner edge the disk becomes optically thick, and a shadow is cast. In fact, if so little matter is present that no shadow would be cast, then the disk would presumably be globally optically thin, and the system would not have been classified as a Herbig Ae/Be star in the first place (it would more resemble a Vega-type star). The second condition is not always met: as our results show, there are situations in which the disk flares, while in other situations it becomes self-shadowed. This depends on how high the surface density (more accurately: optical depth) is at those large radii. If the surface density exceeds a certain threshold, the disk starts to flare. This threshold, however, depends on radius and on the height of the shadow.

If we view these results in terms of the model parameters $M_{\text {disk }}$ and $p$, we can see that there are two ways of arriving at the different solutions. For a given disk mass, the surface density powerlaw will determine the surface densities in the 


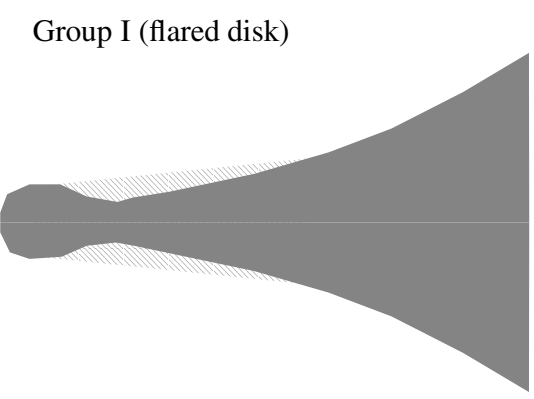

Fig. 8. Pictographic representation of the two disk geometries found.

outer disk. Self-shadowed solutions are then favored by steep powerlaws, which make the rim higher and reduce the surface densities in the outer regions. If on the other hand we keep the surface density powerlaw fixed, and vary the mass, then selfshadowed disks will be favored by low overall disk masses.

\subsection{Dust settling as the cause of self-shadowing}

Another possible way of turning a flaring disk into a selfshadowed disk is dust settling. In this paper we show that the self-shadowing geometry can be found even without invoking dust settling, but it is clear that dust settling will take place and may influence the models. For compact grains with a size of about $0.1 \mu \mathrm{m}$ the settling time scale in the outer regions of a $100 \mathrm{AU}$ disk are of the order of $1 \mathrm{Myr}$. This means that in the typical life time of a disk the dust settles closer to the midplane. Depending on vertical mixing processes this may not proceed all the way to very thin pancake-like disk, but the geometric thickness of the disk can be significantly reduced. If by that time the shadow of the inner rim is still present, such disks would naturally become self-shadowed.

We leave open the question what is more important to produce a self-shadowed disk: disk parameters, or the effect of dust settling. In a forthcoming paper we will model the process of dust settling in detail (Dullemond \& Dominik in prep.).

\subsection{Shadowing and the silicate feature}

The fact that in T Tauri stars and Herbig Ae/Be stars the dust features are generally seen in emission has been explained by earlier models (Calvet 1991, CG97 etc) as arising from the superheated surface layer created by the direct irradiation of the disks's surface by the central star. Naively one would therefore expect that the self-shadowed disks found in this paper would not have emission features, as their surfaces do not receive direct stellar radiation. Yet, as can be seen in Figs. 5-7, even the self-shadowed disks have their silicate feature strongly in emission. There are several reasons for this. First of all, stellar photons can reach the shadowed region by indirect means (Fig. 9). For instance, photons scattering off the upper parts of the puffed-up inner rim may get diverted into the shadowed region, and the thermal near-infrared emission from this upper part of the rim may also irradiate the shadowed region. In fact
Group II (self-shadowed disk)

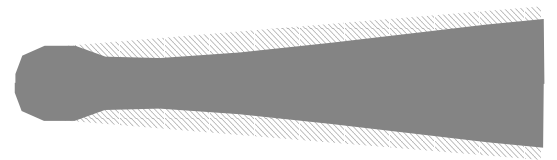

in the models presented here only the latter is at work, since we have neglected scattering.

Another way by which the shadowed region can be heated occurs when the shadowing is imperfect. The A4 model has an almost perfect shadow (see Fig. 3), while the shadowing in the B5 model is clearly less complete (see Fig. 4). This means that even though one cannot really speak of a flaring disk, there is still a tail of the vertical density distribution of the disk that is above the shadow and may therefore produce a dust emission feature.

Finally, when the disk has lost so much of it's small grain content that it becomes optically thin at $7 \mu \mathrm{m}$ and $13 \mu \mathrm{m}$, the silicate feature naturally appears in emission. This in fact plays a role in models B5 and B6.

The indirect heating of the shadowed regions of the disk is crucial, not only for the silicate emission feature, but also for the far-IR flux. If the shadowing were so perfect that no indirect heating were possible, the self-shadowed disks outside of the inner rim would be essentially cold (meaning that they would have the temperature of the surrounding molecular cloud). They would therefore barely produce farIR flux at all. This would be inconsistent with observations of group II sources which clearly do show some far-IR flux, albeit much weaker than that of group I sources. The indirect heating of these self-shadowed regions is responsible for this weak-but-non-negligible far-IR flux, and it is responsible for the fact that our self-shadowed disks of the BL-series have SEDs that are so similar to existing group II sources.

\subsection{Extended emission: PAH features and scattering images}

An important way to study the structure of circumstellar disks in general and self-shadowing in particular is through resolved images. In the sub-mm wavelength regions, all models discussed in this paper will be extended - even though later models of series A will look more compact because most of the disk mass has been moved to the inner regions. A much better test for self-shadowing will be images obtained in near and mid-IR PAH bands, and images obtained in scattered light. PAH emission results from the excitation of PAHs through individual UV photons. Scattered light emerges from the disk when stellar photons in the optical and near-IR are scattered off the disk 


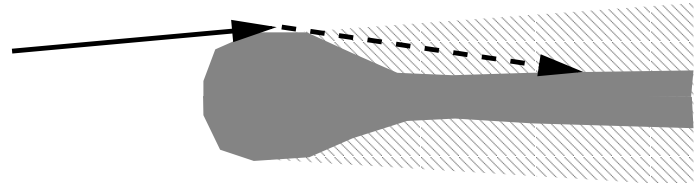

Fig. 9. Pictographic representation of how the shadowed regions of the disk can be irradiated in an indirect way through thermal emission of (and scattering off) the upper part of the puffed-up inner rim. Note that the geometry is exaggerated in this pictogram.

surface into the line of sight. Both processes are not modeled in the current paper, so we cannot quantitatively predict observations. However, since both directly track the illumination of the disk surface with stellar photons (or, on a much lower level, stellar photons scattered towards the disk in the upper regions of the rim), we can make qualitative predictions. If a disk is self-shadowed, both PAH emission and scattered light should be reduced dramatically in the outer disk region, much more so than in a disk which is still flaring but merely has a reduced surface height. In the latter case, the expected reduction factor in the intensity of both PAH and scattered light is only a few while in fully self-shadowed disks it should be orders of magnitude. We note that the models BL4... BL6 are not perfectly self-shadowed - a tenuous tail of the vertical density distribution of the disk still reaches out of the shadow. However, even a small amount of dust settling would be enough to move the available scatterers fully into the shadow. Possible tests for the effect of shadowing in disks therefore include correlating the SED type (group I versus group II) with PAH emission and scattered light images. In group II sources, the PAH emission should be much weaker or even absent - if present it should be compact. Scattered light images of group II sources should be very dim or even completely dark, except at the inner rim. Full studies of these effects are underway, with first results indicating that indeed, group II sources are usually compact (van Boekel et al. 2003) or weak in PAH emission (Acke et al., in preparation). Sources detected so far in scattered light are consistently group I (e.g. Grady et al. 2001; Grady et al. 2000; Danks et al. 2001).

\section{Conclusions}

In this paper we systematically investigated the structure and the SEDs of passive dusty protoplanetary disks around Herbig Ae/Be stars. The models are available in the form of ASCII tables on the internet at the following URL: http://www . mpa-garching . mpg . de/PUBLICATIONS/DATA/ radtrans/flareshadow/

The main conclusions of this paper are as follows:

1. The SEDs of Herbig Ae/Be stars can be quite naturally understood in terms of a dusty circumstellar disk that is passively reprocessing the radiation of the central star. A self-consistent 2D axisymmetric disk model based on 2D continuum radiative transfer and vertical hydrostatic equilibrium seems to be a reasonably accurate description of such a disk.

2. Two kinds of solutions are found: disks with a flaring geometry longwards of about $3 \mathrm{AU}$ and those who are fully self-shadowed by the disk's own puffed-up inner rim. The flaring disks have SEDs that seem in agreement with observed SEDs of group I sources (in the classification scheme of Meeus et al. 2001), while the self-shadowed disks have SEDs similar to those observed from group II sources. These flared and self-shadowed disks are natural solutions of the combined equations of radiative transfer and hydrostatics.

3. The mass distribution within the disk is one important factor in determining whether a disk is flared or selfshadowed. The total mass of the disk is important as well, but may conflict with the observed fact that group II sources are not systematically less massive than group I sources. Keeping the mass fixed, but growing a large fraction of the grains (by coagulation and settling) into a layer of $\mathrm{mm}$ size grains at the midplane does in fact give the right trend from group I to II with a reasonable (sub)mm flux. Moreover, this naturally leads to a less steep (sub)mm slope for group II disks which is indeed observed. Therefore, dust grain growth and settling can be the driving cause of an evolutionary transition turning flaring disks (which appear as group I source) to self-shadowed disks (appearing as group II sources).

4. All disks (both the flaring and the self-shadowed ones) have a $10 \mu \mathrm{m}$ silicate feature in emission. This is in agreement with the known SEDs of group I and group II sources. Only when the disk is seen edge-on, the silicate feature can turn into absorption.

5. The models predict a systematic difference in the strength and extendedness of PAH emission features between group I and group II sources, with the group II sources being weaker than the group I sources. A similar effect is predicted for resolved images of the disks in scattered light: the group II sources being much weaker than the group I sources, perhaps even undetectable.

6. A 2D treatment of radiative transfer is essential in order to find and treat self-shadowed disk solutions.

Acknowledgements. We thank Rens Waters, Antonella Natta, Roy van Boekel, Carol Grady, Mario van den Ancker and Bram Acke for useful discussions and remarks. We would also like to thank the anonymous referee for prompting us to shorten and clarify many aspects of the paper.

\section{References}

Bouwman, J., de Koter, A., van den Ancker, M. E., \& Waters, L. B. F. M. 2000, A\&A, 360, 213

Beckwith, S. V. W., Sargent, A. I., Chini, R. S., \& Guesten, R. 1990, AJ, 99, 924

Bjorkman, J. E., \& Wood, K. 2001, ApJ, 554, 615

Calvet, N., Patino, A., Magris, G. C., \& D’Alessio, P. 1991, ApJ, 380, 617

Chiang, E. 2000, Ph.D. Thesis, California Institute of Technology

Chiang, E. I., \& Goldreich, P. 1997, ApJ, 490, 368 
Chiang, E. I., Joung, M. K., Creech-Eakman, M. J., et al. 2001, ApJ, 547,1077

D’Alessio, P., Calvet, N., \& Hartmann, L. 2001, ApJ, 553, 321

D’Alessio, P., Canto, J., Calvet, N., \& Lizano, S. 1998, ApJ, 500, 411

Danks, A., Vieira, G., \& Grady, C., et al. 2001, AAS, 199, 6014

Dominik, C., Dullemond, C. P., Waters, L. B. F. M., \& Walch, S. 2003, A\&A, 398, 607

Draine, B. T., \& Lee, H. M. 1984, ApJ, 285, 89

Dullemond, C. P. 2000, A\&A, 361, L17

Dullemond, C. P. 2002, A\&A, 395, 853

Dullemond, C. P., Dominik, C., \& Natta, A. 2001, ApJ, 560, 957

Dullemond, C. P., \& Natta, A. 2003, A\&A, 408, 161

Dullemond, C. P., van Zadelhoff, G. J., \& Natta, A. 2002, A\&A, 389, 464

Grady, C. A., Devine, D., \& Woodgate, B., et al. 2000, ApJ, 544, 895

Grady, C. A., Polomski, E. F., \& Henning, T., et al. 2001, AJ, 122, 3396
Malbet, F., \& Bertout, C. 1991, ApJ, 383, 814

Malbet, F., Lachaume, R., \& Monin, J.-L. 2001, A\&A, 379, 515

Mannings, V., \& Sargent, A. I. 1997, ApJ, 490, 792

Meeus, G., Waters, L. B. F. M., Bouwman, J., et al. 2001, A\&A, 365, 476

Miroshnichenko, A., Ivezić, Z., Vinković, D., \& Elitzur, M. 1999, ApJ, 520, L115

Miroshnichenko, A., Ivezic, Z., \& Elitzur, M. 1997, ApJ, 475, L41

Nakazato, T., Nakamoto, T., \& Umemura, M. 2003, ApJ, 583, 322

Natta, A., Prusti, T., Neri, R., Wooden, D., \& Grinin, V. P. 2001, A\&A, 371, 186

Pascucci, I., Wolf, S., Steinacker, J., et al. 2004, A\&A, accepted

van den Ancker, M. E., Bouwman, J., Wesselius, P. R., et al. 2000, A\&A, 357, 325

Waters, L. B. F. M., \& Waelkens, C. 1998, ARA\&A, 36, 233 